DOI: https://doi.org/10.29393/EID3-29CMDE30029

\title{
ESTUDIO DE LA CARGA MENTAL EN PERSONAL DE SALUD DURANTE LA CONTINGENCIA DE COVID-19
}

\author{
STUDY OF MENTAL WORKLOAD IN HEALTH PERSONNEL DURING THE COVID-19 \\ CONTINGENCY
}

\author{
Daniela Rivas-Marquez ${ }^{*}$ \\ Janitsa Lizbeth Ortega-Guerrero** \\ Ernesto Ramírez-Cárdenas ${ }^{* * *}$
}

\begin{abstract}
Resumen: El estudio de los factores psicosociales y de carga mental en los sitios de trabajo siempre han sido de interés para el tomador de decisiones, sin embargo, es a raíz del inicio de la pandemia, ante la aparición del virus COVID-19, que su análisis se ha vuelto indispensable para la calidad de vida del trabajador. El presente estudio busca determinar el nivel de carga mental y las características de sus dimensiones en asistentes médicos, para la generación de propuestas que contribuyan a la mejora de dichos indicadores en una Institución de salud. El procedimiento empleado tiene su fundamento en el método NASA TLX, el cual permite dictaminar la carga mental tanto por niveles y dimensiones. Como resultado se obtuvo que el 50\% (18) del personal de salud presenta carga mental alta y 39\% media; en cuanto a las dimensiones con mayor presencia, se tienen a la frustración con 31\% (11) y rendimiento con 19\% (7); se estratificó la información recabada logrando la identificación de la condición en cada trabajador, así como la generación de propuestas que contribuyen a la mejora de los niveles antes mencionados. En conclusión, se logró recabar información de gran relevancia para la atención de la carga mental en los trabajadores y así contribuir y/o evitar las complicaciones de salud física y mental derivadas de la misma.
\end{abstract}

Palabras clave: Carga mental, NASA TLX, COVID-19.

Abstract: The study of psychosocial factors and mental workload in the workplace has always been of interest to the decision maker, however, it is because of the beginning of the pandemic due to the appearance of the COVID-19 virus that its analysis has become essential for the quality of life of the worker. The present study seeks to determine the level of mental workload and the characteristics of its dimensions in medical assistants for the generation of proposals that contribute to the improvement of these indicators in a health institution. The procedure used is based on the NASA TLX method, which allows the mental workload to be determined by levels and dimensions. As results it was obtained that 50\% (18) of the health personnel present High and 39\% Medium mental workload; as for the dimensions with greater presence we have Frustration with $31 \%$ (11) and Performance 19\% (7); the information collected was stratified achieving the identification of the condition in each worker, as well as the generation of proposals that contribute to the improvement of the levels

\footnotetext{
*Departamento de ingeniería industrial, Instituto Tecnológico de Sonora. Obregón, México. Correo electrónico: daniela.rivas@potros.itson.edu.mx. Orcid: https://orcid.org/oooo-0oo2-0632-9822. Autor de correspondencia.

${ }^{* *}$ Departamento de ingeniería industrial, Instituto Tecnológico de Sonora. Obregón, México. Correo electrónico: janitsa.ortega@potros.itson.edu.mx. Orcid: https://orcid.org/oooo-0oo1-9066-5898

*** Departamento de ingeniería industrial, Instituto Tecnológico de Sonora. Obregón, México. Correo electrónico: eramirez@itson.edu.mx. Orcid: https://orcid.org/oooo-0oo2-5248-724X
} 
mentioned above. In conclusion, it was possible to collect information of great relevance for the attention of the mental workload in workers and thus contribute and/or avoid the physical and mental health complications derived from it.

Keywords: Mental load, NASA TLX, COVID-19.

Recepción: 27.10.2021 / Revisión: 04.11.2021 / Aceptación: 08.12.2021

\section{Introducción}

Se le llama coronavirus a la familia de agentes patógenos que ha provocado diferentes enfermedades tanto en animales como humanos (Redacción conecta, 2020, 19 de marzo). El coronavirus SARS-COV-2 es un virus que apareció en China. Después se extendió a todos los continentes del mundo provocando una pandemia. Actualmente Europa y América son los más afectados (Coronavirus México, 2021).

De acuerdo con la figura 1 "línea del tiempo", el 7 de diciembre de 2019 China comunicó que un gran grupo de personas en Wuhan poseen neumonía, siendo el comienzo de una pandemia. Después, el 13 de enero de 2021 el mismo país comparte públicamente la secuencia genética de COVID-19, y para finales del mes la Organización Mundial de la Salud (OMS) declara emergencia sanitaria. El 11 de marzo de 2021 la OMS declara al COVID-19 como una pandemia global. Se puede observar que el 13 de marzo del 2020 la OMS asegura que Europa es el nuevo epicentro de la pandemia. Tres meses más tarde los contagios aumentan a diez millones y a medio millón de muertes en el mundo. El 2020 termina con más de 84 millones de contagios en el mundo y para el 7 de julio de 2021 el mundo alcanza los cuatro millones de fallecidos por COVID-19.

Figura 1. Línea del tiempo.

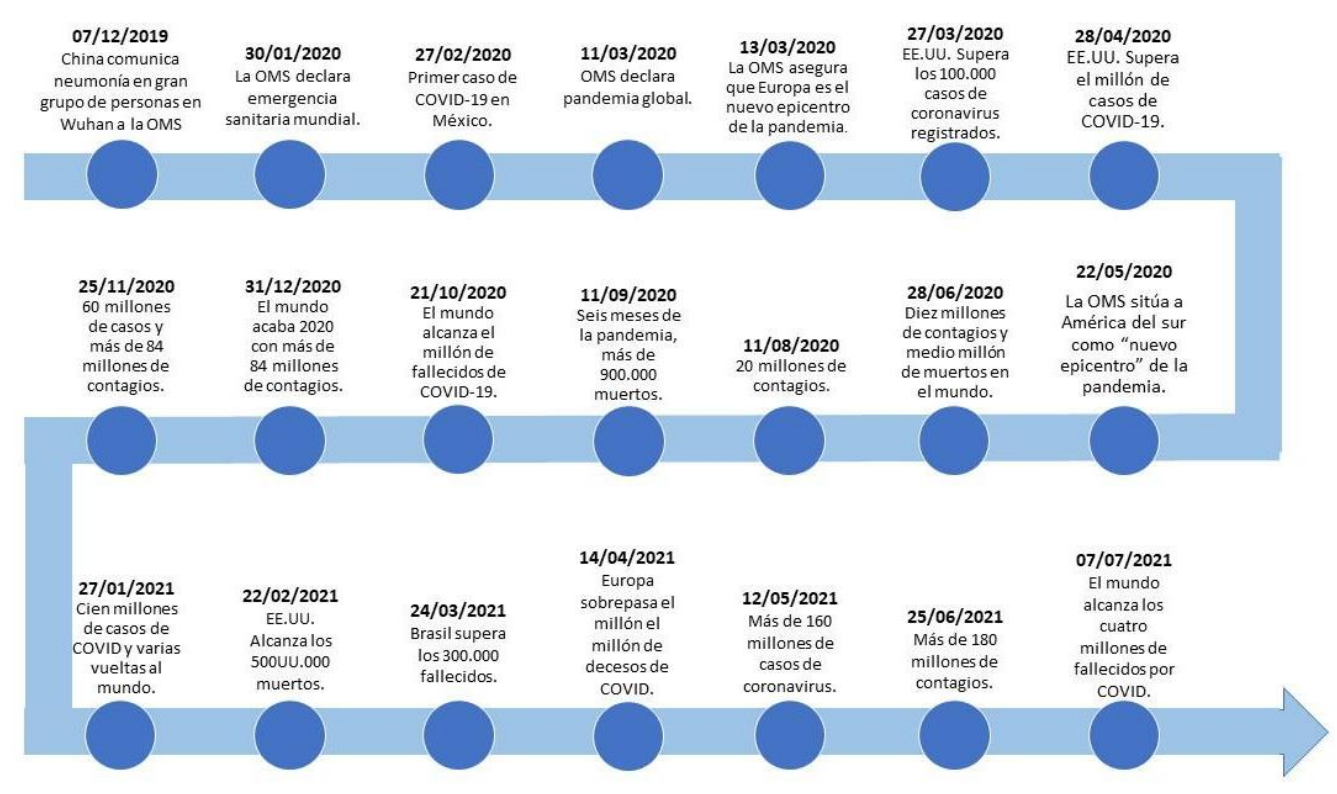

Fuente. Noticias en España RTVE (2021). 
La pandemia del SARS-COV-2 aunque se tenga una mortalidad desigual en cada país, en todo el mundo ha dejado más de cuatro millones y medio de muertes. Los países con más defunciones por COVID-19 son Estados Unidos, India y Brasil, la comparación entre las muertes de países con más de un millón de habitantes en relación con su población demuestra que el país con una tasa de mortalidad alta es Perú debido a que por cada 100.000 habitantes se cuentan con más de 600 decesos. En el caso de España, reporta actualmente una tasa de 180 defunciones, anteriormente este país se encontraba en el segundo lugar en este ranking y en la actualidad no está ni siquiera en los primeros 20 países con mayor mortalidad.

En la siguiente figura se aprecian los continentes de color rojo (América), azul (Europa), verde (Asia) y naranja (África). En base al tamaño de color de la imagen, se muestra la cantidad de casos y muertes durante la pandemia. En América el primer lugar es Estados Unidos con 42.931.354 casos y 688.032 muertes, el país con más contagios y muertes de Europa es Rusia, el cual cuenta con 7.313.112 y 200.245 respectivamente. En Asia, India cuenta con 33.652.745 casos y 446.918 muertes. Por último, en Sudáfrica, país del continente de África, se cuenta con 2.896.943 casos y 87.052 muertos.

Figura 2. Casos y muertes por coronavirus a nivel mundial.

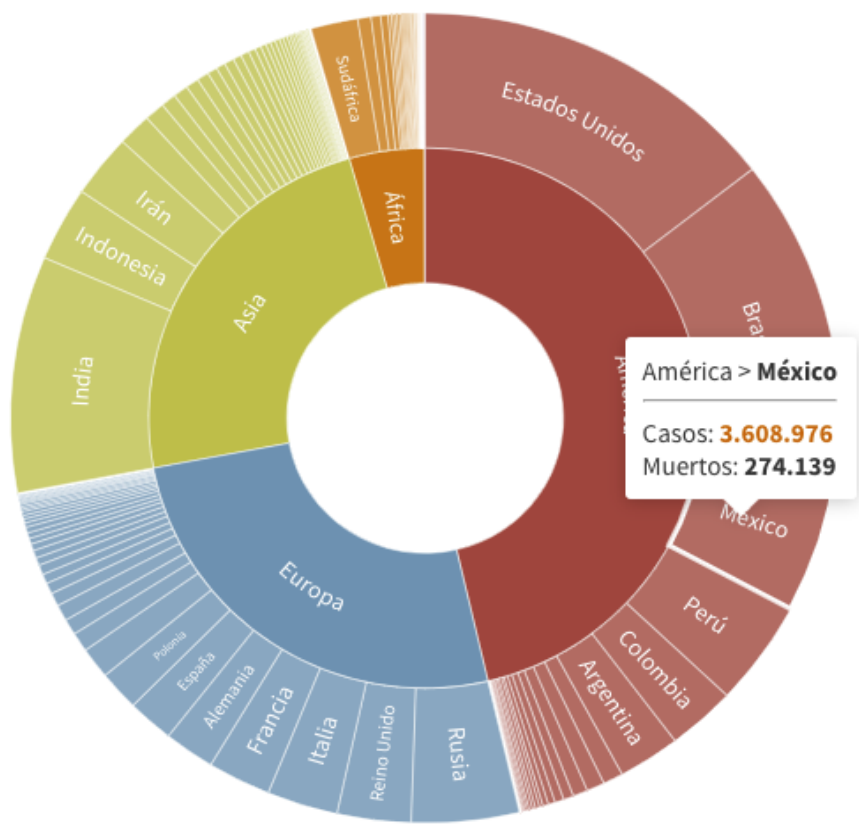

Fuente. Noticias en España RTVE (2021).

En diciembre de 2019 apareció SARS-COV-2 en el Medio Oriente lo cual desencadenó la enfermedad a la que hoy conocemos como COVID-19, su extensión llegó a todos los rincones del mundo aterrorizando a la población y, finalmente, siendo declarada una pandemia global por la Organización Mundial de la Salud (OMS) en el año 2020. Desde que el COVID-19 fue declarado pandemia, se han producido varios cambios en la comunidad médica mundial y los profesionales de la salud se enfrentan a nuevos desafíos y situaciones nuevas sin precedentes (Díez, 2020). En la actualidad en México se tienen 3.608.976 casos confirmados y 274.129 defunciones por COVID-19. 
En el mapa de la figura 3 se muestra el semáforo epidemiológico actual en México, el cual permite observar con facilidad el nivel de contagio de cada estado. Según la Secretaría de Salud (2021), el nivel más alto es el rojo, en la segunda quincena de mayo ningún estado contaba con ese color, esto significa evitar salir si no es estrictamente necesario, se permiten solo las actividades económicas esenciales. Los estados los cuales deben tener más precaución están de color naranja, el cual significa que la operación de actividades económicas y sociales serán con un aforo del 50\%, además de reducción de movilidad comunitaria. El color amarrillo quiere decir que la operación de actividades económicas y sociales serán con un aforo del $75 \%$ y una disminución ligera en el espacio público. Por último, los estados de color verde no tienen restricciones de movilidad, el modelo educativo funcionará bajo la nueva normalidad de acuerdo a lo establecido por la SEP y la operación de actividades económicas y sociales se llevarán a cabo de manera habitual.

Figura 3. Semáforo epidemiológico en México.

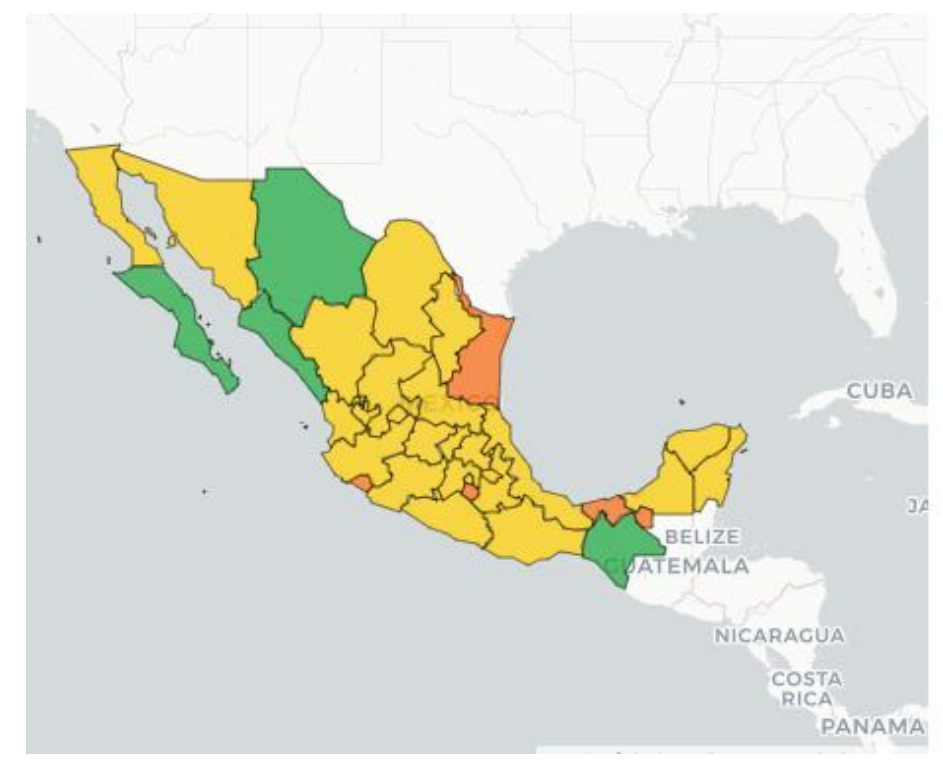

Fuente. Coronavirus México (2021).

Derivado de los contagios, la ocupación hospitalaria incrementó de forma abrupta generando un aumento en la carga de trabajo de los trabajadores hospitalarios en todas las áreas establecidas. Un año después de su inicio, en el mes de marzo de 2021, la ocupación hospitalaria aumentó del 11 al 17\% (Gobierno del Estado de Sonora, 2021, 22 de marzo).

Las actividades económicas a inicios de la pandemia del COVID-19 fueron directamente impactadas por las estrategias de contención sanitarias, lo que generó una crisis económica. Esto provocó que las empresas a nivel mundial sufrieran de falta de ingresos debido a un menor índice de consumo. Dependiendo de la industria, el tiempo máximo estimado que una empresa puede operar sin liquidez ronda entre uno y tres meses. Considerando la estimación del tiempo de recuperación de una crisis económica como un factor multiplicador de la duración, es decir, si la crisis llegara a durar 3 meses, la recuperación tardaría 9 meses adicionales a la duración de la crisis. Hasta ahora se estima que la crisis pueda impactar entre un 5\% y un 10\% los estimados de crecimiento económico de los países (Meade, 2020, abril). 
En el primer año, según Cortes-Meda \& Ponciano-Rodríguez (2021, 4 de mayo) se registraron alrededor de 151 muertes de COVID-19 por cada 100.000 habitantes, y esta enfermedad se ha posicionado como la primera causa de muerte, dejando atrás a las enfermedades cardiovasculares y la diabetes mellitus.

Durante el primer año de la pandemia de COVID-19, la figura a continuación muestra las comorbilidades más comunes en los casos positivos, las cuales han sido hipertensión (17\%), obesidad (15\%), diabetes (13\%), tabaquismo (7\%) y otras enfermedades (8\%); mientras que en aquellos que fallecieron por esta causa, las comorbilidades más comunes fueron hipertensión (45\%), diabetes (37\%), obesidad (22\%), enfermedad cardiovascular (11\%), tabaquismo (8\%) e inmunosupresión (5\%). Las personas que tuvieron dos o más comorbilidades incrementaron el riesgo de morir. El 28\% no padecía comorbilidad (CortesMeda \& Ponciano-Rodríguez, 2021, 4 de mayo).

Figura 4. Principales comorbilidades en defunciones positivos a COVID-19.

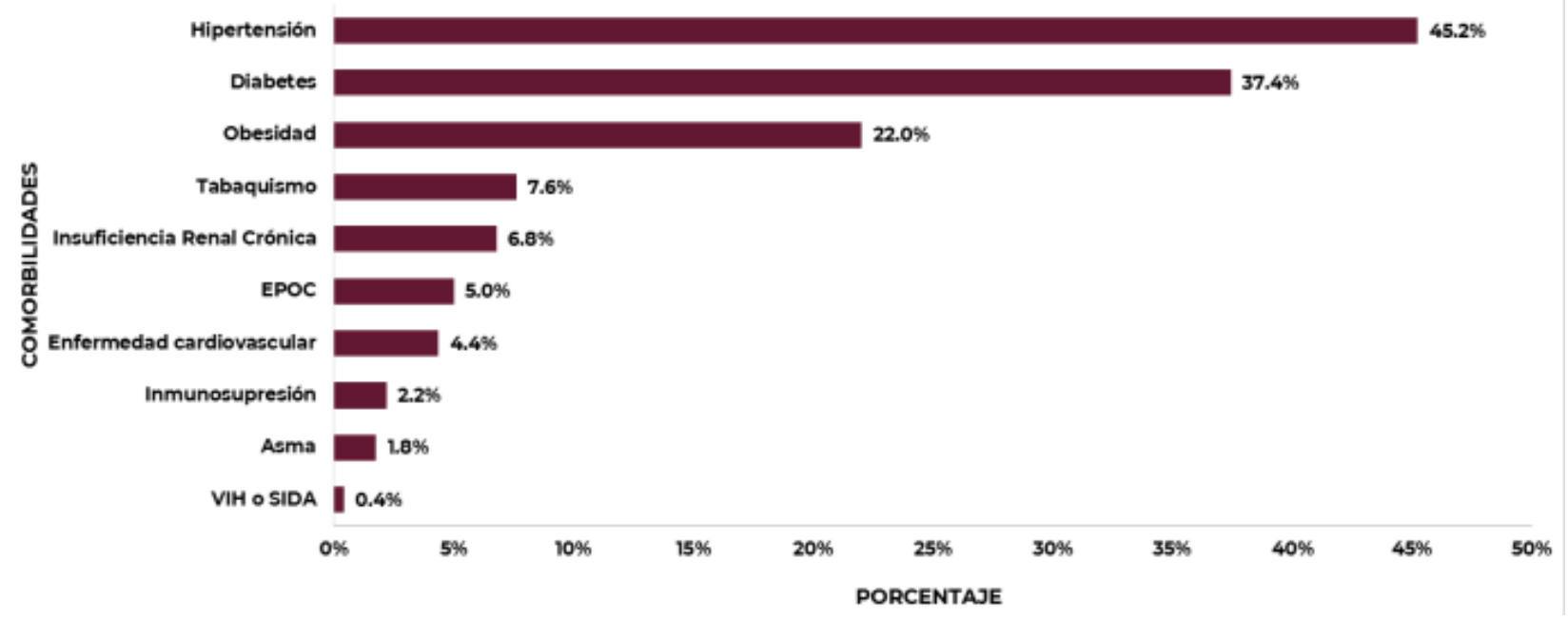

Fuente. Cortes-Meda y Ponciano-Rodríguez (2021, 4 de mayo).

Del total de contagios (2.144.588) en 2020, únicamente el 25\% recibió atención hospitalaria. A nivel nacional, solo una de cada cinco personas fallecidas por COVID-19 fue atendida en una Unidad de Cuidados Intensivos (UCI). El contraste más significativo es el del IMSS, que únicamente proporcionó terapia intensiva al $4 \%$ de las personas fallecidas que atendió. En relación con la intubación, procedimiento que debería de aplicarse a la mayoría de los casos graves, solo han sido intubadas el $29 \%$ de las personas fallecidas. De nuevo, el máximo contraste se observa en el IMSS, en cuyas unidades médicas solamente se entubó al 20\% de las personas fallecidas. Esto es extremadamente relevante, porque el IMSS atiende a una de cada tres personas con COVID-19 (Hernández-Bringas, 2021, 7 de enero).

Debido a la reclusión y la minimización de las actividades comerciales y el transporte debido al estado de alerta, ha supuesto una disminución de las emisiones de gases de efecto invernadero, sobre todo de la industria y del transporte por carretera. En el corto plazo, esto es una buena noticia para la consecución de los objetivos de mitigación de gases de efecto invernadero, la contaminación atmosférica en las ciudades se ha reducido e incluso cabría esperar que este hecho redunde en la salud de las personas que habitan estos núcleos, 
normalmente sometidas a altos niveles de contaminación (Sanz, 2020, 7 de abril).

La carga mental se define como el número de procesos requeridos para terminar una actividad de acuerdo al tiempo en que una persona puede encontrar las respuestas en su memoria (Mondelo et al., 2000). Con la aplicación de nuevas tecnologías, los trabajos requieren cada vez más que los trabajadores procesen la información. Al tener tanta presión, el personal de salud se ve sumergido a una alta serie de demandas que, tras ser valoradas como estresores, provocan un desequilibrio sistémico tal como se muestra en la figura 5 .

Figura 5. Grupos de factores que configuran la carga de trabajo mental y sus efectos.

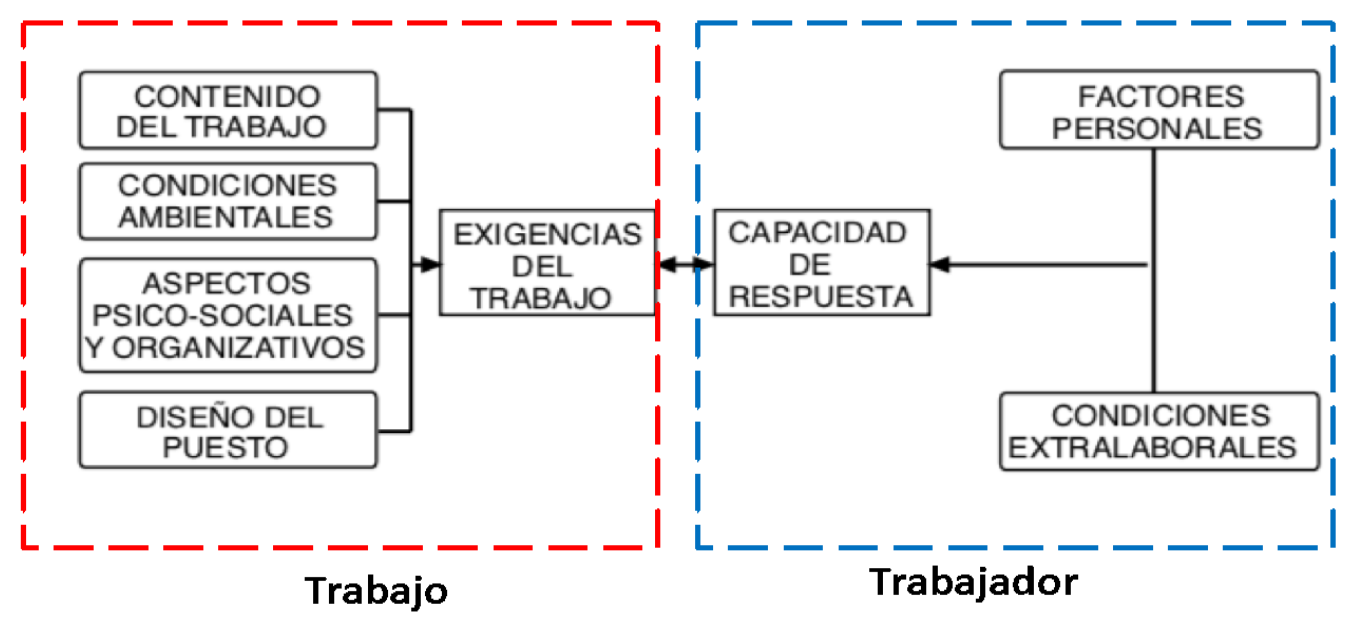

Fuente. García et al. (2002).

En la figura anterior se muestran los factores de carga mental tales como:

- Contenido del trabajo: exigencias propias de la tarea.

- Condiciones ambientales: ruido, vibraciones, iluminación, temperatura, etc.

- Psicosociales y de organización: ritmo de trabajo, la duración de la jornada, el número, la duración y la distribución de las pausas, y otros factores como las relaciones laborales, las posibilidades de comunicación, el estilo de mando de los jefes, etc.

- Diseño del puesto: adaptación del mobiliario y del espacio físico, y el grado de comodidad o incomodidad.

Además, con respecto al trabajador, es importante contemplar la edad, estado de salud, grado de fatiga, nivel de activación y variaciones en el nivel de vigilancia, agudeza sensorial y rapidez perceptiva, nivel y tipo de inteligencia, nivel de aprendizaje y experiencia en la tarea, características de personalidad (ansiedad, introversión/extroversión, etc.), y actitud hacia la tarea, motivación, interés por la tarea, satisfacción, etc. (García et al., 2002).

Por lo general, estos estresores son considerados por los trabajadores, por la cantidad de trabajo y cuidados personales al realizar sus tareas, siendo el rendimiento el aspecto más afectado por el personal de salud al realizar sus actividades.

La plantilla laboral se compone de alrededor de 232 médicos, 583 enfermeros, 36 
asistentes médicos, entre personal administrativo y de limpieza. Para atender la demanda de atención en esta región se cuenta con 188 camas, 11 quirófanos, una unidad de cuidados intensivos de adultos, una coronaria y una de cirugía cardiovascular. En un solo día se brindan 388 consultas, de las cuales 359 son de especialidad, se realizan 20 intervenciones quirúrgicas, y se practican 3.115 análisis clínicos. Diariamente egresan 22 personas y se internan 21, por lo que la ocupación hospitalaria es del 91 por ciento.

Las jornadas laborales son de 8 horas con 2 días de descanso a la semana, en caso de turnos nocturnos son 11,4 horas 3 veces a la semana. Aunque las jornadas laborales antes de la pandemia no eran tan largas, después de la pandemia la institución de salud solicitaba turnos extraordinarios y se gratifican como horas extras, lo que provocó que el cansancio y carga mental se elevará.

La situación generada en los hospitales por efecto de la pandemia por COVID-19 caracterizada por el aumento en el número de pacientes, la disminución en la disponibilidad de recursos materiales y el incremento en el número de contagios ha provocado un mayor porcentaje de casos de agotamiento en el personal que labora en dichas instituciones (tabla 1).

Tabla 1. Indicadores de salud.

\begin{tabular}{|c|c|c|}
\hline Indicador & Antes de la pandemia & $\begin{array}{c}\text { Durante la contingencia } \\
\text { de la pandemia }\end{array}$ \\
\hline Número pacientes & 170 & 188 \\
\hline Recursos materiales & $80 \%$ & $65 \%$ \\
\hline Número de contagios & 0 & 47 \\
\hline Porcentaje de casos de agotamiento & $15 \%$ & $40 \%$ \\
\hline
\end{tabular}

Los trabajadores de la salud han visto cómo sus colegas durante la pandemia fueron infectados por el COVID-19, siendo incapaces de brindarles atención humanitaria debido a la sobrecarga de trabajo, y otros se convirtieron en víctimas de agresiones o estigmas sociales. Dichos aspectos, sumados a los indicadores antes mencionados, afectan la salud mental de estos profesionales presentando en ellos un mayor grado de estrés, ansiedad y depresión reduciendo su capacidad de concentración, comprensión y toma de decisiones (Ruiz-Frutos \& Gómez-Salgado, 2021).

Se ha observado que los trabajadores de la salud toman decisiones complicadas en su día a día, dichas decisiones incluyen asignar recursos escasos a pacientes igualmente necesitados y una búsqueda constante sobre cómo brindar atención a todos los pacientes gravemente enfermos. Esto puede causar que algunos trabajadores experimenten problemas de salud mental que se ven agravados con sus propias necesidades de equilibrar su salud física y mental con la de los pacientes, familiares y amigos. A raíz del inicio de contagio por SARS-COV-2 los profesionales de salud han tenido un impacto psicológico importante, cerca del 40\% sufre de algún tipo de agotamiento ya sea físico o emocional, por lo que, aumentó el requisito de atención psicológica en trabajadores hospitalarios (Ruiz-Frutos \& GómezSalgado, 2021). 
Ante la situación descrita se contempla la siguiente pregunta: ¿Cuál será el nivel de carga mental y sus principales dimensiones en trabajadores de una institución de salud?

El objetivo de este estudio es determinar el nivel de carga mental y las características de sus dimensiones en trabajadores para la generación de propuestas que contribuyan a la mejora de los indicadores de una institución de salud.

\section{Materiales y métodos}

Los participantes del estudio abarcaron al personal de salud con categoría de asistente médico. La metodología se basó en la aplicación del método NASA TLX, cuyos pasos se muestran a continuación.

1. Determinar el tamaño de la muestra a estudiar: Para la determinación de la muestra a estudiar se consideró un 95\% de confianza y un 5\% de error. Para el estudio se optó por considerar al total de población y/o personal de salud.

2. Recolectar datos: Para esta actividad se aplicó una encuesta utilizando el instrumento del método NASA TLX de Hart y Staveland (1988), considerando como tareas a todas aquellas actividades que realizan diariamente en su trabajo. Previo a su aplicación se le explicó a los participantes en qué consiste cada una de las dimensiones que conforman el método y la importancia del estudio.

3. Analizar e interpretar los datos: Se utilizó una base de datos realizada en Microsoft Excel para la captura de la información adquirida, y una vez realizada la captura de datos se realizó el análisis estadístico determinando los porcentajes de incidencia de las diferentes variables. Se presentaron dichos resultados de manera ordenada a través de gráficas, y se indagó sobre los principales motivos de la carga mental de las asistentes.

\section{Resultados y discusión}

Como fase inicial, una vez calculado el 95\% de confianza y un $5 \%$ de error, se determinó que la cantidad de encuestas aplicadas serían a 36 asistentes médicos. El siguiente paso fue caracterizar la muestra cuyos datos relevantes se enlistan a continuación:

- El 22\% de las trabajadoras encuestadas se encuentran entre los 24 a 27 años de edad, el $17 \%$ tienen 28 años de edad, el $25 \%$ se encuentran entre 31 y 34 años de edad y el resto se encuentra entre 35 a 48 años.

- Con relación al estado civil de los encuestados, el 53\% es soltera y el 47 \% es casada.

- Con respecto a la escolaridad de los encuestados, el 72\% tiene estudios universitarios, $22 \%$ preparatoria y el resto educación secundaria.

Una vez aplicadas las encuestas y obtenidos los datos relevantes, se evaluaron los resultados organizando los datos y observando las dimensiones sobresalientes de la carga mental del personal de salud bajo estudio. 
En la figura 6 se muestra que el 50\% del personal de salud bajo estudio se encuentra en un nivel de carga mental alto, esto es debido a que se ha verificado que el entorno laboral tiene un fuerte impacto en la salud mental de los trabajadores. El 39\% se encuentra en el nivel medio de carga mental, mientras que un $11 \%$ tiene un nivel bajo de carga mental, tomando en cuenta que el $41,8 \%$ son menores de 30 años de edad, siendo trabajadores jóvenes. La pandemia del COVID-19 expuso a los trabajadores sanitarios a situaciones en las cuales tenían que tomar decisiones complejas trabajando bajo presión en situaciones de alto estrés.

Figura 6. Nivel de carga mental.

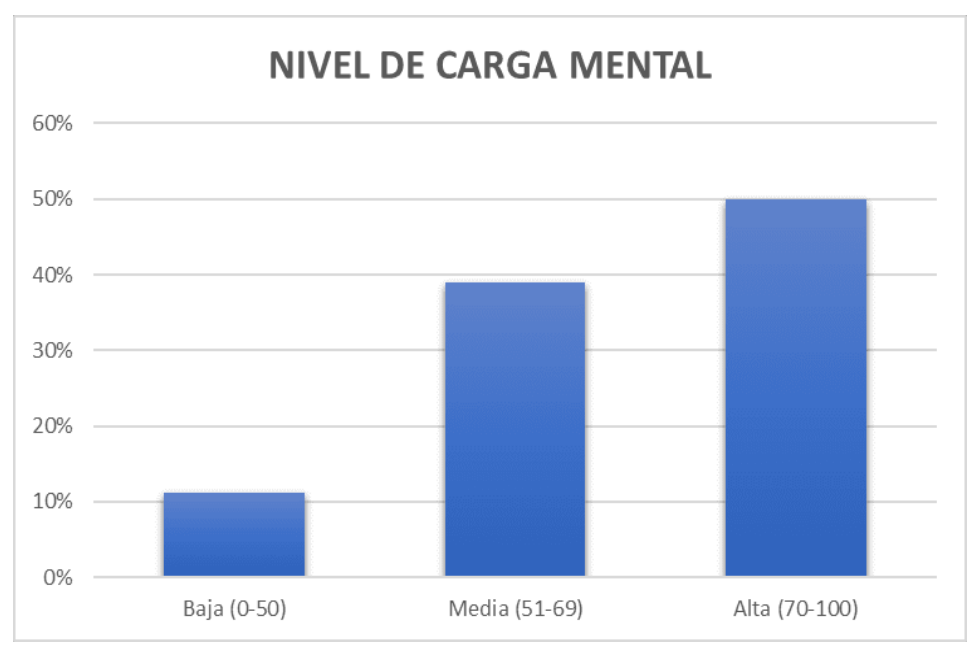

En el personal evaluado como se muestra en la figura 7, las dimensiones más elevadas son el rendimiento y la frustración, con un porcentaje de 30\% correspondiente a 11 personas en cada dimensión. La exigencia mental tuvo un resultado de $19 \%$ con un total de 7 y el resto recaen en exigencia física y esfuerzo.

Figura 7. Porcentaje por tipo de dimensión seleccionada.

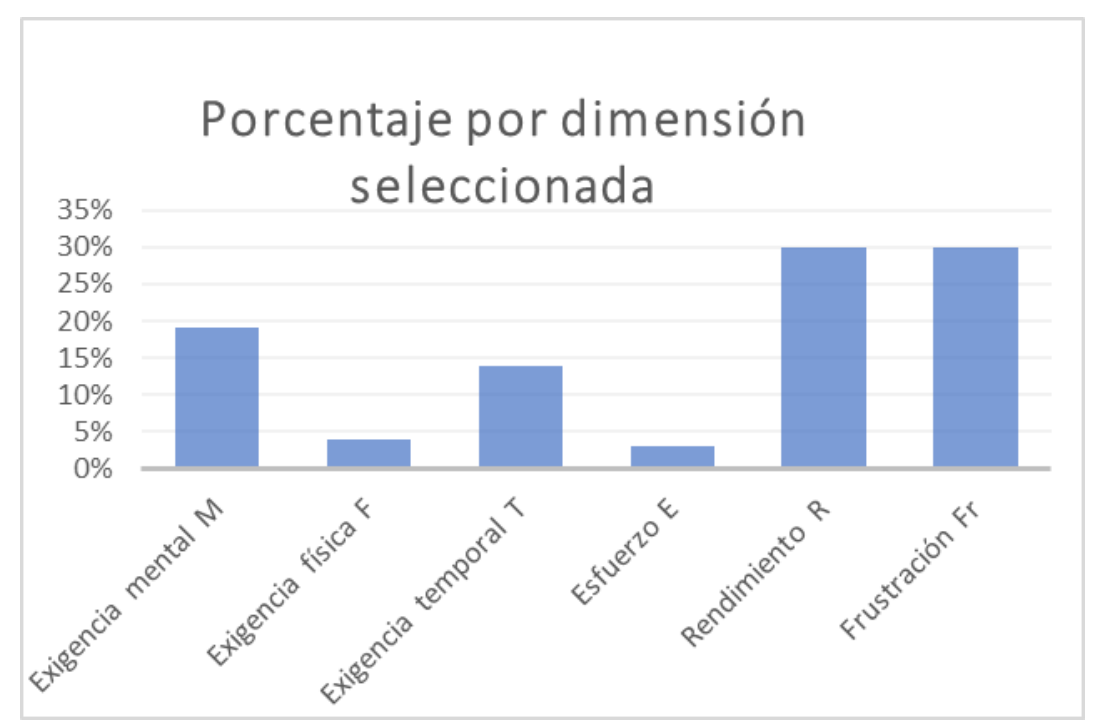

En la tabla 2 se muestran los resultados del estudio. Las celdas de color verde claro significan que el riesgo es bajo (o-50 puntos); solo 4 trabajadoras tienen un riesgo bajo. El 
riesgo medio (51-69 puntos) es el color amarillo; 14 trabajadoras son de riesgo medio. Al evaluar las 36 trabajadoras, 18 tienen un riesgo alto (70-100 puntos), por lo que es necesario actuar.

Tabla 2. Resultados del estudio.

\begin{tabular}{|c|c|c|c|}
\hline Trabajadora & $\begin{array}{c}\text { Dimensión } \\
\text { más alta }\end{array}$ & $\begin{array}{c}\text { Nivel } \\
\text { de } \\
\text { carga } \\
\text { mental }\end{array}$ & Riesgo \\
\hline 1 & Fr & 60 & Medio \\
\hline 2 & $\mathrm{M}$ & 55 & Medio \\
\hline 3 & $\mathrm{Fr}$ & 89 & Alto \\
\hline 4 & $\mathrm{Fr}$ & 81 & Alto \\
\hline 5 & $\mathrm{R}$ & 61 & Medio \\
\hline 6 & $\mathrm{M}$ & 88 & Alto \\
\hline 7 & $\mathrm{~T}$ & 54 & Medio \\
\hline 8 & $\mathrm{Fr}$ & 83 & Alto \\
\hline 9 & $\mathrm{~T}$ & 54 & Medio \\
\hline 10 & $\mathrm{~T}$ & 54 & Medio \\
\hline 11 & $\mathrm{M}$ & 29 & Bajo \\
\hline 12 & $\mathrm{~T}$ & 61 & Medio \\
\hline 13 & $\mathrm{R}$ & 74 & Alto \\
\hline 14 & $\mathrm{R}$ & 70 & Alto \\
\hline 15 & $\mathrm{Fr}$ & 80 & Alto \\
\hline 16 & $\mathrm{Fr}$ & 73 & Alto \\
\hline 17 & $\mathrm{R}$ & 44 & Bajo \\
\hline 18 & $\mathrm{R}$ & 57 & Medio \\
\hline
\end{tabular}

\begin{tabular}{|c|c|c|c|}
\hline Trabajadora & $\begin{array}{c}\text { Dimensión } \\
\text { más alta }\end{array}$ & $\begin{array}{c}\text { Nivel } \\
\text { de } \\
\text { carga } \\
\text { mental }\end{array}$ & Riesgo \\
\hline 19 & $\mathrm{Fr}$ & 73 & Alto \\
\hline 20 & $\mathrm{M}$ & 91 & Alto \\
\hline 21 & $\mathrm{~T}$ & 73 & Alto \\
\hline 22 & $\mathrm{E}$ & 32 & Bajo \\
\hline 23 & $\mathrm{M}$ & 51 & Medio \\
\hline 24 & $\mathrm{Fr}$ & 69 & Alto \\
\hline 25 & $\mathrm{R}$ & 85 & Alto \\
\hline 26 & $\mathrm{R}$ & 46 & Bajo \\
\hline 27 & $\mathrm{Fr}$ & 91 & Alto \\
\hline 28 & $\mathrm{M}$ & 93 & Alto \\
\hline 29 & $\mathrm{~F}$ & 55 & Medio \\
\hline 30 & $\mathrm{R}$ & 65 & Medio \\
\hline 31 & $\mathrm{Fr}$ & 82 & Alto \\
\hline 32 & $\mathrm{M}$ & 64 & Medio \\
\hline 33 & $\mathrm{R}$ & 65 & Medio \\
\hline 34 & $\mathrm{R}$ & 63 & Medio \\
\hline 35 & $\mathrm{Fr}$ & 85 & Alto \\
\hline 36 & $\mathrm{R}$ & 72 & Alto \\
\hline
\end{tabular}

La frustración de los trabajadores de la salud se elevó significativamente desde los inicios de la pandemia, debido a que se tenía que trabajar a un ritmo diferente, lo que provocó tensión e inquietud con respecto a las medidas de seguridad que se requerían, como lo es la distancia mayor a 1,50 metros, el uso de cubrebocas y el lavado frecuente de manos. Para el personal de salud al comenzar la pandemia era complicado mantener todos los factores de seguridad cuando se tenía contacto directo con pacientes positivos COVID-19, debido a que aún no se contaba con equipo de protección necesario y mucho menos una vacuna que los protegiera, esto provocaba la preocupación de llevar la enfermedad a sus seres queridos. Algunos durante meses se alejaban completamente de sus familias para evitar los contagios.

En la figura 8 se muestran dos mapas sinópticos. En uno se observa el nivel de carga mental dividido en si es alta, media o baja, para después señalar los trabajadores que sufren 
cada uno. En el siguiente se muestran las dimensiones que son frustración, rendimiento, exigencia mental, exigencia temporal, esfuerzo y exigencia física, en cada una se observan los trabajadores que indican en esa dimensión, además de identificar en color rojo a los de carga mental alta, esto para un mayor enfoque a la hora de establecer las propuestas de mejora.

Figura 8. Estratificación por nivel de carga mental y por dimensión.

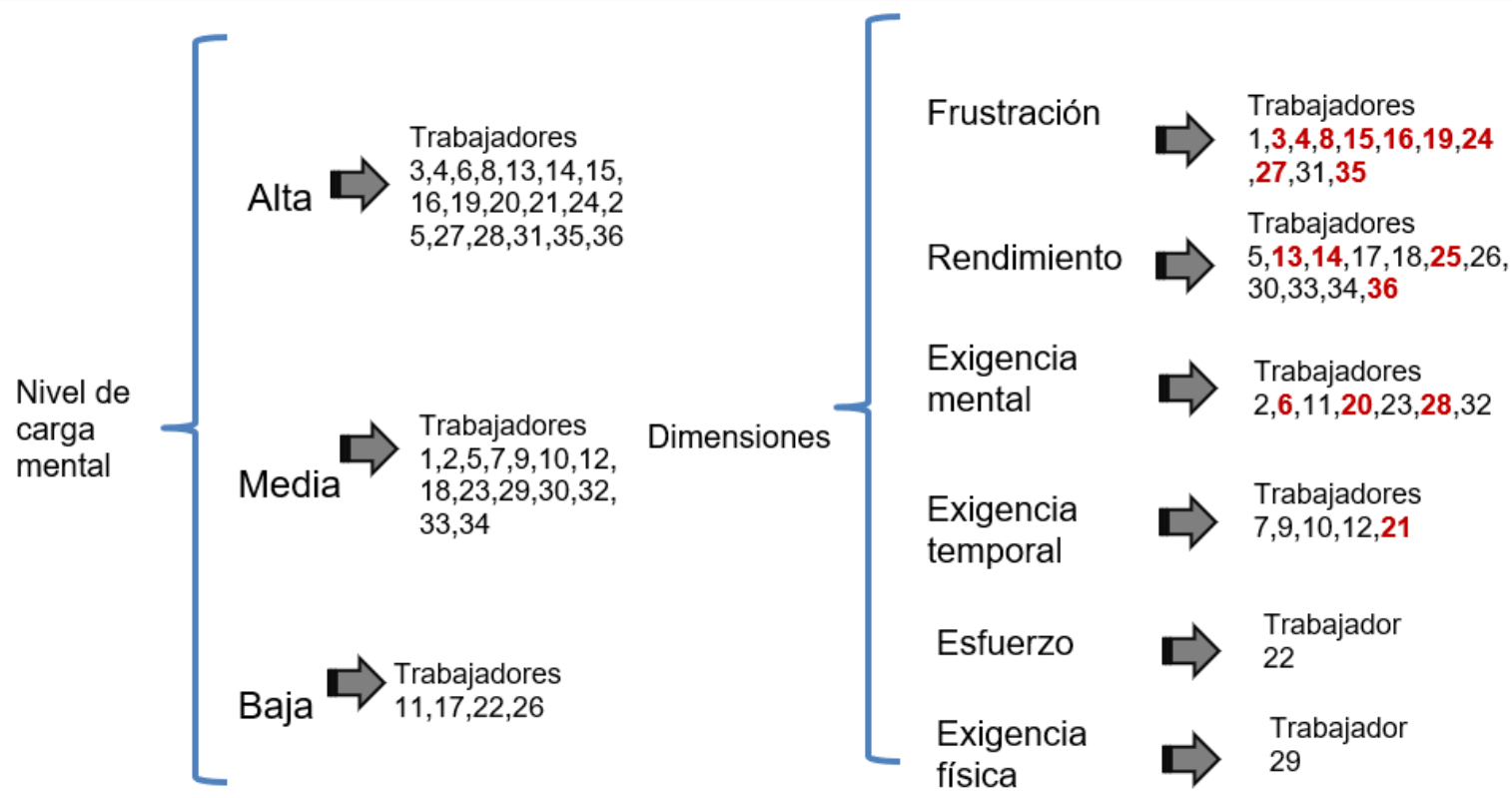

Una vez identificados a los trabajadores, por nivel de carga y dimensión, se presentan las recomendaciones que se mencionan en la tabla 3.

Tabla 3. Plan de atención por cada dimensión.

\begin{tabular}{|c|l|}
\hline Dimensión & \multicolumn{1}{c|}{ Acciones } \\
\hline Frustración & $\begin{array}{l}\text { Establecer límites claros en el trabajo, promover los hábitos saludables, } \\
\text { y buscar estrategias para contrarrestar el estrés y la frustración. }\end{array}$ \\
\hline \multirow{5}{*}{ Rendimiento } & $\begin{array}{l}\text { Es necesario destacar que el éxito de los programas en general no es la } \\
\text { corrección, sino la prevención, así como el fortalecimiento de la propia } \\
\text { capacidad de resistencia, ya que cada persona tiene una capacidad de } \\
\text { resistencia diferente, la cual se modula dependiendo de la edad, sexo, } \\
\text { entre otras, así como con hábitos saludables tanto físicos como } \\
\text { mentales. } \\
\text { Una reestructuración de plazas en la institución de salud podría ayudar } \\
\text { en el rendimiento del personal, ya que las actividades que se realizan } \\
\text { serán bien distribuidas entre ellos, otorgando una mejor atención a los } \\
\text { pacientes, además de menos carga de trabajo, mayor eficiencia y } \\
\text { eficacia. }\end{array}$ \\
\hline Exigencia mental & $\begin{array}{l}\text { Mantener una comunicación directa con empleados y sindicatos acerca } \\
\text { de la forma en la que la pandemia está afectando el trabajo. }\end{array}$ \\
\hline Exigencia temporal & $\begin{array}{l}\text { Reorganizar el tiempo de trabajo (tipo de jornada, duración, flexibilidad, } \\
\text { etc.) y facilitar suficiente margen de tiempo para la auto distribución de } \\
\text { algunas breves pausas durante cada jornada de trabajo. }\end{array}$ \\
\hline
\end{tabular}




\begin{tabular}{|c|l|}
\hline \multirow{2}{*}{ Esfuerzo } & $\begin{array}{l}\text { Actualizar los útiles y equipos de trabajo (manuales de ayuda, listas de } \\
\text { verificación, registros y formularios, procedimientos de trabajo, etc.) } \\
\text { siguiendo los principios de claridad, sencillez y utilidad real. }\end{array}$ \\
\hline \multirow{2}{*}{ Exigencia física } & $\begin{array}{l}\text { Capacitación a los trabajadores para facilitar y agilizar la atención hacia } \\
\text { el paciente. } \\
\text { Rediseñar el lugar de trabajo, adecuando espacios, iluminación, } \\
\text { ambiente sonoro, etc. }\end{array}$ \\
\hline
\end{tabular}

Además de lo anterior, se recomienda que a los trabajadores con carga mental alta se les apoye a través de la realización de talleres de interés con temas relacionados a factores psicosociales, y se les otorgue apoyo psicológico enfocado de inicio a atender los altos niveles de frustración y rendimiento.

\section{Conclusiones}

Al utilizar el método de evaluación NASA TLX se obtuvo que la carga mental de la mitad del personal de salud evaluado es alta, dando por hecho el estrés que viven día con día por la pandemia del COVID-19. Se demostró que las dimensiones más altas en el estudio es el rendimiento y la frustración al realizar sus actividades.

Debido a que el $47,2 \%$ del personal evaluado se encuentra casado y con familia, además de la carga mental derivada de las actividades de su trabajo, también cuentan con asuntos personales, los cuales la incrementan. El realizar este tipo de estudio permite identificar qué tan propensos son a padecer estrés generado por la carga mental. Así mismo, brinda información sobre las diferentes dimensiones en las que se debe trabajar para minimizar esta carga (carga mental, carga física, exigencia temporal, frustración, rendimiento y esfuerzo).

El conocer la carga mental a la que son sometidos el personal de salud, permitirá también a los departamentos involucrados generar programas o estrategias que contribuyan a disminuir este factor, repercutiendo en la reducción del rezago o incluso en la deserción, ya que este podría conducir a un mayor aprovechamiento durante su jornada laboral, y además a prevenir problemas de salud tanto física como mental. 


\section{Referencias}

Coronavirus México. (2021). Coronavirus. Gobierno de México. https://coronavirus.gob.mx/informacion-accesible/

Cortes-Meda, A., \& Ponciano-Rodríguez, G. (2021, 4 de mayo). Impacto de los determinantes sociales de la COVID-19 en México. Boletín COVID-19 Salud Pública, 2(17), 9-13. http://dsp.facmed.unam.mx/wp-content/uploads/2013/12/COVID-19-No.17-O4-Impactode-los-determinantes-sociales-de-la-COVID-19-en-Me\%CC\%81xico.pdf

Díez, F. (2020). Origen del SARS-COV-2. Instituto de salud Carlos III.

García, O. S., Del Hoyo-Delgado, M. A., Centro Nacional de Nuevas Tecnologías, \& Instituto Nacional de Seguridad e Higiene en el Trabajo. (2002). La carga mental de trabajo. Instituto Nacional de Seguridad e Higiene en el Trabajo. https://www.insst.es/documents/94886/96076/carga+mental+de+trabajo/2fd91b55-f1914779-be4f-2c893c2ffe37

Gobierno del Estado de Sonora. (2021, 22 de marzo). Actualiza Mapa Sonora Anticipa municipios en riesgo por COVID-19. http://salud.sonora.gob.mx/acciones/boletinesinformativos/actualiza-mapa-sonora-anticipa-municipios-en-riesgo-por-covid-19.html

Hart, S. G., \& Staveland, L. E. (1988). Development of NASA-TLX (Task Load Index): Results of empirical and theoretical research. Advances in Psychology, 52, 139-183. https://doi.org/10.1016/So166-4115(08)62386-9

Hernández-Bringas, H. (2021, 7 de enero). Índice COVID-19 en México: Un perfil sociodemográfico.

CEPAL.

https://repositorio.cepal.org/bitstream/handle/11362/46557/2000528 LDN111 05 Hernandez.pdf?sequence $=1 \&$ isAllowed $=\mathrm{y}$

Meade, G. L. (2020, abril). La crisis del COVID-19. EY. https://www.ey.com/es mx/covid-19/lacrisis-del-covid-19

Mondelo, P. R., Gregori, E., \& Barrau, P. (2000). Ergonomia 1. Fundamentos. Alfaomega.

Noticias en España RTVE. (2021). Casos y muertes globales por coronavirus. https://www.rtve.es/noticias/20210924/mapa-mundial-del-coronavirus/1998143.shtml

Redacción conecta. (2020, 19 de marzo). Pandemia, cepa y otras palabras sobre el coronavirus. Conecta. https://tec.mx/es/noticias/nacional/salud/pandemia-cepa-y-otras-palabras-sobreel-coronavirus-que-debes-saber

Ruiz-Frutos, C., \& Gómez-Salgado, J. (2021). Efectos de la pandemia por COVID-19 en la salud mental de la población trabajadora. Archivos de Prevención de Riesgos Laborales, 24(1), 611. https://doi.org/10.12961/aprl.2021.24.01.01

Sanz, A. (2020, 7 de abril). El coronavirus y el planeta. National Geographic. https://www.nationalgeographic.com.es/ciencia/coronavirus-y-futuro-planeta 15406

Secretaría de Salud. (2021). Lineamiento para la estimación de riesgos del semáforo por regiones COVID-19. https://coronavirus.gob.mx/wp-content/uploads/2021/o8/2021.8.18Metodo semaforo COVID.pdf 